\title{
Topographie 3D satellite (Pléïades) : Application à l'évolution côtière de la Langue de Barbarie
}

\author{
Adélaïde TAVENEAU ${ }^{1}$, Rafael ALMAR ${ }^{1}$, Erwin BERGSMA ${ }^{1,3}$, \\ Abdoulaye NDOUR ${ }^{2}$, Boubou ALDIOUMA SY ${ }^{3}$, Solange LEMAI-CHENEVIER ${ }^{4}$, \\ Anne LIFERMANN ${ }^{4}$, Alain GIROS ${ }^{4}$, Maxime GOSSELIN ${ }^{5}$, Thierry GARLAN ${ }^{5}$
}

1. LEGOS, OMP - UMR 5566 (CNES-CNRS-IRD-Université de Toulouse), 14 Avenue Edouard Belin, 31400 Toulouse, France.

adelaide.taveneau@legos.obs-mip.fr ; rafael.almar@ird.fr ;

erwin.bergsma@legos.obs-mip.fr

2. Université Cheikh Anta Diop, BP 5005, Dakar-Fann, Sénégal. abdoulayndour75@yahoo.fr

3. Université Gaston Berger,

Nationale 2, route de Nagllèle, BP : 234, Saint Louis, Sénégal.

bouboualdiouma@yahoo.fr

3. CNES - UMR 5566 (CNES-CNRS-IRD-Université de Toulouse)

18 Avenue Edouard Belin, 31400 Toulouse, France.

solange.lemai-chenevier@cnes.fr ; anne.liferlann@cnes.fr ; alain.giros@cnes.fr

4. SHOM Brest Département géologie marine, 29240 Brest, France.

maxime.gosselin@shom.fr ; thierry.garlan@shom.fr

\section{Résumé :}

La compréhension de la morphologie est limitée par le manque de continuité entre l'échelle locale (vidéo, drone), et l'échelle régionale : les nouvelles technologies peuventelles répondre à ce besoin? La ville de Saint Louis, située à l'embouchure du Fleuve Sénégal, et séparée de l'Atlantique par la flèche sableuse de la Langue de Barbarie, représente un lieu phare de la vulnérabilité littorale liée aux changements climatiques, et aux enjeux socio-économiques sur la scène internationale. Les satellites Pléïades (CNES/Airbus) ont une résolution de $1 \mathrm{~m}$ rééchantillonnée à $0.5 \mathrm{~m}$, et peuvent couvrir une zone de $350 \mathrm{~km} * 20 \mathrm{~km}$. Une reconstruction topographique à partir de ces clichés est donc réalisable par méthode de tri-stéréogrammétrie, avec le logiciel Ames Stéréo Pipeline (ASP) développé par la NASA. Des séquences rafales (vidéo) de trois à dix images de la Langue de Barbarie ( $\mathrm{dT} \sim 8 \mathrm{~s}$ ) sont acquises tous les 6 mois, et nous offrent une vision inégalée de la dynamique sédimentaire de la zone. Cette méthodologie a déjà montré des résultats prometteurs (ALMEIDA et al., 2019) lors de son application sur la côte Aquitaine.

\section{Mots-clés :}

Topographie, Pléïades, Erosion, Satellites, Stéréogrammétrie. 


\section{Thème 2 - Dynamique sédimentaire}

\section{Introduction}

Avec plus de $50 \%$ de la population mondiale résidant à moins de $100 \mathrm{~km}$ des côtes, le littoral est une zone de conflits permanents entre les phénomènes naturels, et les interventions humaines. Souvent exploitée à des fins agricoles, industrielles, ou touristiques, et sans cesse soumis à l'action des processus marins, la bande littorale est aujourd'hui exposée à des pressions environnementales et économiques sans précédent. Dans un contexte de réchauffement climatique, et plus particulièrement de la montée du niveau de la mer, la protection des littoraux représente un enjeu socio-économique majeur.

Les plages sableuses (31\% des littoraux à l'échelle mondiale) sont des systèmes à la dynamique complexe, et $24 \%$ d'entre elles s'érodent à des vitesses alarmantes de plus de $0.5 \mathrm{~m} / \mathrm{an}$ (LUIJENDIJK et al., 2018). L'accès aux paramètres haute résolution topographiques et bathymétriques d'un littoral est primordial afin de pourvoir faire face aux nombreux problèmes sociétaux actuels (GESCH et al., 2016), et permet l'implémentation de modèles pouvant prédire l'évolution côtière (ALMEIDA et al., 2019). C'est pourquoi des méthodes de suivi de la côte ont été mis en place au travers des années, afin de pouvoir mieux comprendre les mécanismes et procédés de la dynamique d'une plage. Les techniques d'acquisition vidéo permettent de collecter des données hydrodynamiques, et morphologiques d'une plage, à haute fréquence, à échelle locale. Cependant, elles ne permettent pas de visualiser la dynamique d'un littoral à échelle régionale.

Dans ce contexte, les acquisitions satellitaires Pléíades (CNES/Airbus) offrent la possibilité d'observer à haute résolution la dynamique d'un littoral à grande échelle, en utilisant une méthode de tri-stéréogrammétrie pour une reconstitution $3 \mathrm{D}$ de la topographie. Cette méthode, à l'aide d'une séquence d'images panchromatiques Pléïades, a déjà été utilisée pour estimer la topographie de Capbreton sur la côte Aquitaine $(0.35 \mathrm{~m} \leq \mathrm{RMSE} \leq 0.48 \mathrm{~m}$; ALMEIDA et al., 2019).

\section{Matériel et méthodes}

\subsection{Site d'étude : Saint Louis}

La ville de Saint Louis (Sénégal, figure1b) se situe à l'embouchure du Fleuve Sénégal, et est séparée de l'océan Atlantique par une flèche sableuse : la Langue de Barbarie (figure 1b). Ce site est exposé à de fortes pressions environnementales : lieu d'une puissante dérive littorale due au régime de vagues obliques, et de submersions hydrologiques fréquentes, la Langue de Barbarie est soumise à une très forte érosion. De plus, à la suite d'une importante inondation, une brèche a été creusée dans la Langue de Barbarie en 2003, faisant 4 mètres de large à l'origine. Sa largeur est d'aujourd'hui de plusieurs kilomètres avec un taux d'érosion moyen de -3.72 m/an (NDOUR et al., 2018). 


\section{XVİ̀mes Journées Nationales Génie Côtier - Génie Civil \\ Le Havre 2020}

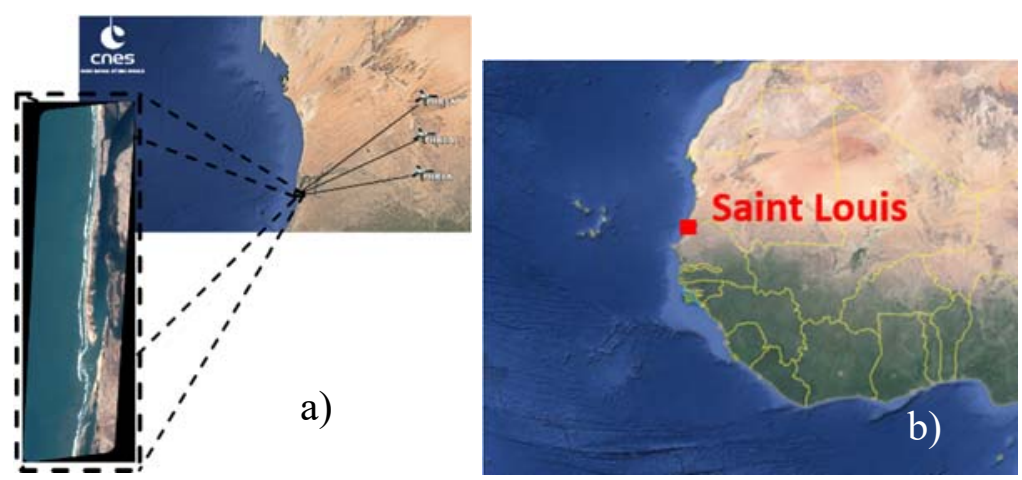

Figure 1. a) Clichés satellites de la plage de Saint Louis. b) Site d'étude : Saint Louis.

\subsection{Données satellites}

Une séquence de 12 images (Multispectrales et Panchromatiques) en rafales (dT 8s) a été acquise en Mars 2019, ainsi qu'une séquence de 3 images en Septembre 2019. Leur résolution est de $1 \mathrm{~m}$ rééchantillonnée à $0.5 \mathrm{~m}$. Ce sont les images panchromatiques qui ont été utilisées pour effectuer la tri-stéréo de cette étude, car la résolution horizontale du modèle digital d'élévation (DEM) est meilleure qu'avec les images multispectrales (COLLIN et al., 2018). La tri-stéréo est réalisée avec le logiciel Ames Stereo Pipeline développé par la NASA (SHEAN et al., 2016), qui procède par évaluation de la corrélation point par point de deux paires d'images pour une tri-stéréo, puis par combinaison des résultats de ces deux corrélations. Les DEM obtenus ont par la suite été masquées avec la méthode Channel Colour Divergence (CDD), basée sur le ratio des bandes couleur d'une image (TURNER et al., 2001 ; ALMAR et al., 2012), puis corrigées verticalement à l'aide de GCPs (Ground Control Points). Trois images satellites ont été utilisées pour reconstruire la topographie du site d'étude : respectivement $\mathrm{dT} \sim 8 \mathrm{~s}$ avant l'image la plus au Nadir, l'image la plus au Nadir, et $\mathrm{dT} \sim 8 \mathrm{~s}$ après l'image la plus au Nadir (figure 2).

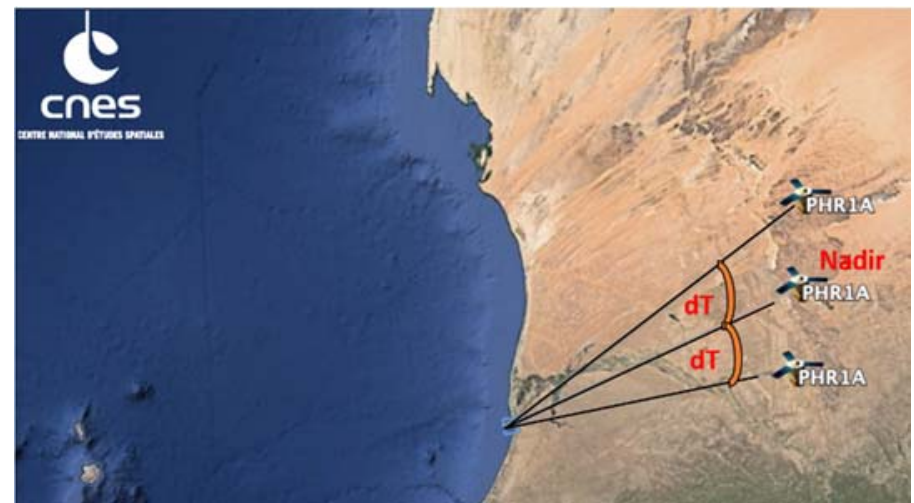

Figure 2. Clichés satellites utilisés pour la reconstruction topographique par stéréogrammétrie. 


\section{Thème 2 - Dynamique sédimentaire}

\subsection{Levés topographiques}

Le jeu de données topographiques utilisé dans cette étude a été collecté en Mars 2019 par le SHOM avec un Real Time Kinematic GPS (RTK-GPS) de résolution centimétrique.

\section{Résultats}

\subsection{La reconstruction topographique par tri-stéréogrammétrie}

Trois images panchromatiques, espacées de $\mathrm{dT} \sim 8 \mathrm{~s}$ chacune, sont utilisées pour réaliser la reconstruction topographique de la Langue de Barbarie (figure 3). L'acquisition d'images Pléïades tous les 6 mois permet de mettre en évidence la dynamique de la côte : on peut s'apercevoir du fort recul du trait de côte au Sud de la flèche sableuse en comparant les figures 3a, et 3b, acquises respectivement en Mars 2019 et Septembre 2019.
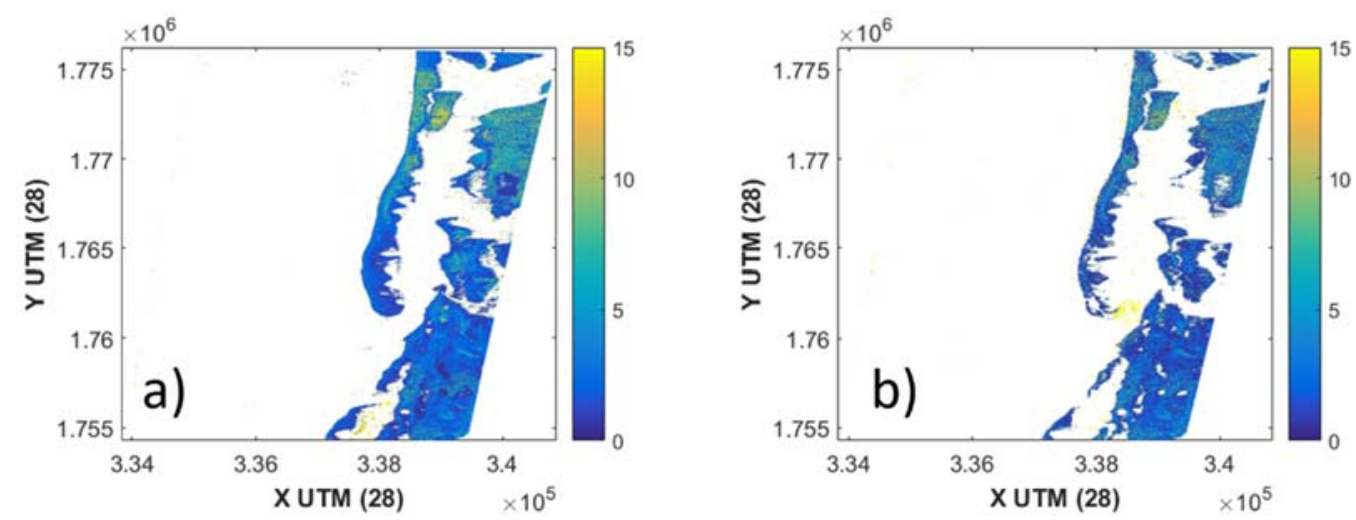

Figure 3. a) Reconstruction topographique de la Langue de Barbarie - mars 2019.

b) Reconstruction topographique de la Langue de Barbarie - septembre 2019.

\subsection{Masque sur les données}

Afin de filtrer les données, un masque leur a été appliqué consistant à créer un masque de l'eau pour enlever les étendues d'eau et la mer. La construction de celui-ci se base sur le ratio des couleurs de l'image brute satellite acquise (multispectrale) : les bandes rouge, verte et bleue ont des valeurs similaires sur le sable, alors que leur valeurs divergent dans l'eau (ALMAR et al., 2012). C'est le masque obtenu avec le ratio des bandes rouge et verte qui a été retenu, et utilisé dans cette analyse (figure 4).

Toutefois cette méthode a des limites : lorsque l'image d'origine comporte des sédiments en suspension dans l'eau (figure 4b), le masque les détecte et ne permet pas une correction optimale des données. C'est pourquoi un masque itératif a été mis en place : dès qu'une acquisition Pléïades a lieu, un nouveau masque est calculé et vient compléter les précédents. 


\section{XVI'̀mes Journées Nationales Génie Côtier - Génie Civil \\ Le Havre 2020}
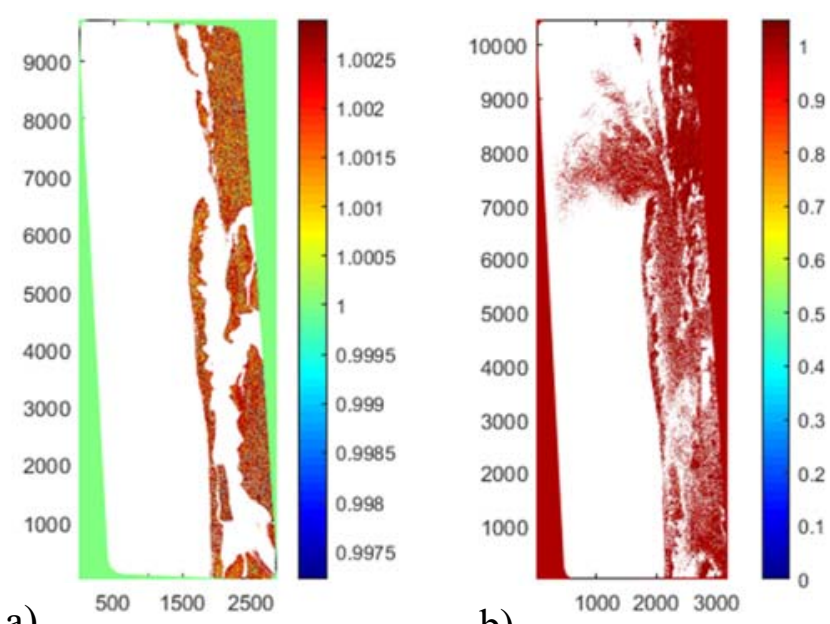

b)

Figure 4. (a) Masque ratio rouge/vert sur les données de Mars 2019. (b) Masque ratio rouge/vert sur les données Septembre 2019.

\subsection{Analyse de 1'erreur}

La carte de corrélation (figure 5) de la tri-stéréo réalisée par ASP permet d'évaluer la qualité de la reconstitution topographique : plus la corrélation entre les trois images d'origines est grande, et meilleure est la topographie de sortie.

En chaque point, la corrélation entre les trois images de sortie est calculée : celle-ci est faible dans l'océan $(<0.2$, figure 5$)$ car il n'y a pas/peu de correspondance entre ces pixels pour chaque image d'entrée. Le même constat que pour le masque peut être fait : lorsqu'il y a un fort transport de sédiments à l'embouchure du fleuve Sénégal (figure 5b), la corrélation des trois images d'entrée dans l'embouchure est élevée.

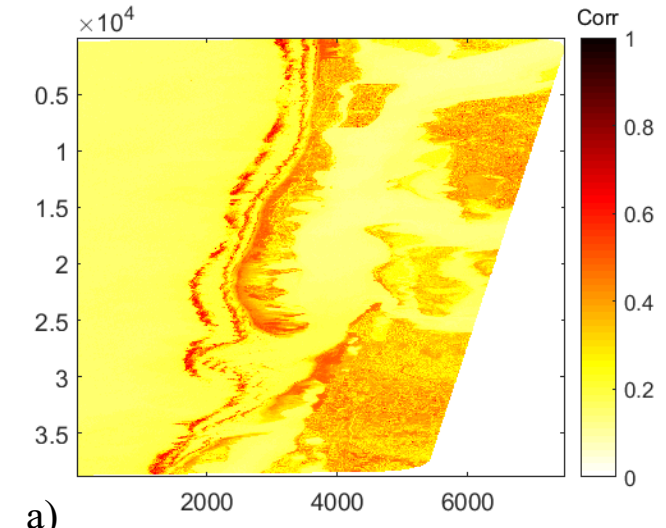

a)

Figure 5. (a) Carte de corrélation de la tri-stéréo des images panchromatiques de Mars 2019. (b) de Septembre 2019.

\section{Conclusions}

Dans ce travail, les résultats obtenus montrent le potentiel de l'utilisation des satellites pour l'observation de la dynamique sédimentaire, car ils permettent de couvrir une échelle 


\section{Thème 2 - Dynamique sédimentaire}

plus large que les méthodes de suivi du littoral classiques, et apportent une compréhension plus globale de la morphodynamique d'une côte. Cependant, un transport sédimentaire important peut empêcher une reconstitution optimale de la topographie car la méthode ne distingue plus la terre de la mer. Les topographies obtenues par méthode de tristéréogrammétrie seront d'autant plus précises que la résolution de la séquence d'images utilisées pour la reconstruction est élevée : elle est un facteur limitant de cette précision, et de ce fait, non négligeable.

\section{Références bibliographiques}

ALMAR R., RANASINGHE R., SENECHAL N., BONNETON P., ROELVINK D., BRYAN K.R., MARIEU V., PARISOT J-P. (2012). Video-based detection of shorelines at complex meso-macro tidal beaches. Journal of Coastal Research, Vol. 28(5), pp 1040-1048. https://doi.org/10.2112/JCOASTRES-D-10-00149.1

ALMEIDA L., ALMAR R., BERGSMA E., BERTHIER E., BAPTISTA P., GAREL E., DADA O., ALVES B. (2019). Deriving high spatial-resolution coastal topography from sub-meter satellite stereo imagery. Remote Sensing, 11, 590. https://doi.org/10.3390/rs11050590 COLLIN A., HENCH J., PASTOL Y., PLANES S., THIAULT L., SCHMITT R., HOLBROOK S., DAVIES N., TROYER M. (2018). High resolution topobathymetry using Pleiades-1 triplet: Moorea Island in 3D. Remote Sensing of Environment, Vol. 208, pp 109-119. https://doi.org/10.1016/j.rse.2018.02.015

GESCH D.B., BROCK J.C., PARRISH C.E., ROGERS J.N., WRIGHT C.W. (2016). Introduction: special issue on advances in topobathymetric mapping, models, and applications. J. Coast. Res., Vol. 76 (sp1), pp 1-3. https://doi.org/10.2112/SI76-001

LUIJENDIJK A., HAGENAARS G., RANASINGHE R., BAART F., DONCHYTS G., AARNINKHOF S. (2018). The state of world's beaches. Sci Rep 8, 6641, https://doi.org/10.1038/s41598-018-24630-6

NDOUR A., LAÏBI R., SADIO M., DEGBE C. (2018). Management strategies for coastal erosion problems in west Africa: Analysis, issues, and constraints drawn from the example of Senegal and Benin. Ocean \& Coastal Management, Vol 156, pp 92-106. https://doi.org/10.1016/j.ocecoaman.2017.09.001

SHEAN D.E., ALEXANDROV O., MORATTO Z.M., SMITH B.E., JOUGHIN I.R., PORTER C., MORIN P. (2016). An automated, open-source pipeline for mass production of digital elevation models (DEMs) from very-high-resolution commercial stereo satellite imagery. ISPRS J. Photogramm. Remote Sens, Vol. 116, pp 101-117. https://doi.org/10.1016/j.isprsjprs.2016.03.012

TURNER I., LEYDEN V., SYMONDS G., MCGRATH J., JACKSON A., JANCAR T., AARNINKHOF S.G.J., ELSHOFF I.E. (2001). Comparison of observed and predicted coastline changes at the gold coast artificial (surfing) reef, Sydney, Australia. In: Edge, B.L. (ed.), Coastal Engineering 2000, Proc. of the $27^{\text {th }}$ Intl. Conference on Coastal Engineering, Sydney, Australia, ASCE, pp 1836-1847. https://doi.org/10.1061/40549(276)143 